\title{
Germline and gonosomal mosaicism in the ATR-X syndrome
}

\author{
Satvinder Bachoo and Richard J Gibbons \\ Nuffield Department of Clinical Biochemistry and Cellular Science, University of Oxford, John Radcliffe Hospital, \\ Headington, Oxford, UK
}

\begin{abstract}
We have identified two females who are mosaic for an $A T R X$ mutation. One case, in whom the mutation was undetectable in peripheral blood and buccal cells, has two affected sons and is therefore presumed to be a germline mosaic. In another case, the $A T R X$ mutation is weakly detectable in the peripheral blood but only one of her three children who share the diseaseassociated haplotype carries the mutation and therefore it is concluded that she is a gonosomal mosaic. These cases provide the first molecular evidence for the occurrence of post-zygotic mutation in $\mathrm{X}$-linked $\alpha$ thalassaemia mental retardation syndrome. The possibility of germline mosaicism must therefore be considered in the genetic counselling of ATR-X families.
\end{abstract}

Keywords: ATR-X syndrome; XLMR; $A T R X$ mutation; germline mosaicism

\section{Introduction}

$\mathrm{X}$ linked $\alpha$ thalassaemia mental retardation syndrome (ATR-X) is a rare disorder characterised in male patients by severe mental retardation, a typical facial appearance, genital abnormalities and $\alpha$ thalassaemia. ${ }^{1}$ In this X-linked recessive disorder, the biological fitness in affected individuals is zero and, as expected, new mutations are frequently encountered in affected families. Therefore, when counselling the parents of sporadic cases and assessing the risk of recurrence, the ability to identify female carriers is of great importance.

In ATR-X syndrome, female carriers are intellectually normal. Donnai et $a l^{2}$ reported hypertelorism and midface hypoplasia in a mother and daughter who were proven carriers. However, in over 70 other families

Correspondence: Dr Richard Gibbons, Nuffield Department of Clinical Biochemistry and Cellular Science, University of Oxford, John Radcliffe Hospital, Headington, Oxford, OX3 9DU, UK. E-mail: rgibbons@immsvr.jr2.ox.ac.uk Fax: + 441865 221834; Tel: + 441865220483

Received 8 March 1999; revised 8 June 1999; accepted 18 June 1999 there is no indication that facial changes or any other associated phenotypic abnormalities are present in female carriers (unpublished data). Nevertheless, in approximately $25 \%$ of obligate carriers, rare cells with $\mathrm{HbH}$ inclusions (the hallmark of $\alpha$ thalassaemia) may be found. ${ }^{1}$ Studies of the pattern of $\mathrm{X}$ inactivation in carriers for ATR-X show that the mutant X chromosome is predominantly inactivated in cells from a variety of tissues, explaining the scarcity of $\mathrm{HbH}$ inclusions in these individuals. ${ }^{3}$ The presence of $\mathrm{HbH}$ inclusions and a highly skewed pattern of $\mathrm{X}$ inactivation has, in the past, been used to identify female carriers in affected families. The former test, however, lacks sensitivity and the latter specificity as $5-10 \%$ females in general population have skewed $\mathrm{X}$ inactivation. Characterisation of the underlying mutation greatly facilitates the identification of carriers and if the mother is negative for the mutation the residual risk depends on the likelihood of germline mosaicism.

ATRX, the gene mutated in ATR-X patients, has been identified. ${ }^{4}$ Characterisation of the underlying mutation in affected boys has allowed 'definitive' testing of females in 60 families. This has revealed two females who are mosaic for the ATRX mutation. One 
case, in whom the mutation was undetectable in the somatic tissues tested, has two affected sons and is therefore presumed to be a germline mosaic. In another case the $A T R X$ mutation is weakly detectable in the peripheral blood but only one of her three children who share the disease-associated haplotype carries the mutation and therefore it is concluded that she is a gonosomal mosaic, ie both somatic and germline mosaicism is present. These observations allow an estimate to made of the frequency of germline mosaicism in ATR-X syndrome.

\section{Patients and Methods}

\section{Families}

The affected males in the families described have the classical features associated with ATR-X syndrome (pedigree 11 (Figure 1 ) and pedigree 19 (Figure $2^{1}$ ). In pedigree 19 , two males $(2-1,2-4)$ were born prematurely and died soon after birth. No further clinical information is available.

\section{Haplotype Analysis}

Polymorphic markers DXS559, DXS227, DXS1225, DXS986 and DXS995 (details via Genome Database http:/ /www.gdb.org) were analysed by PCR and polyacrylamide electrophoresis.

\section{Mutation Analysis}

The mutations in pedigrees 11 and 19 have been described previously. ${ }^{5}$ The numbering adopted in this report is based on U75653; the addition of eight bases at the 5' end corrects this to the transcriptional start site. ${ }^{6}$ In pedigree 11 the mutation 961G $>$ A destroys a StyI restriction site CCTTGG and creates an $\mathrm{MboI}$ restriction site GATC. Genomic DNA from family members was amplified by PCR using primers xnp127 (5'-ATCCATCGCTCGAAACTCGG-3') and PHD1 (5'-AGCCGTGACTCAGATGGAATGGATG-3'). Aliquots of the product were digested either with StyI or MboI (New England Biolabs (UK) Ltd, Hitchin, Hertfordshire, UK) and the fragments resolved by electrophoresis in $1 \%$ agarose.

In pedigree 19 the mutation $(934 \mathrm{G}>\mathrm{T})$ was detected using allele-specific oligonucleotide hybridisation. Oligonucleotide probes were designed to hybridise preferentially to wildtype or mutant sequence as described in. ${ }^{7}$ Genomic DNA from family members was amplified by PCR using primers xpn103 (5'-TATTGTGGACAACTCCTTTCGACC-3') and xnp126 (5'-TCCTGGAGATTTCCCAG-3'). The PCR fragments were electrophoresed on $1 \%$ agarose and blotted onto Hybond- $\mathrm{N}^{+}$membrane (Amersham Life Science Ltd, Little Chalfont, Buckinghamshire, UK). Oligonucleotides (wildtype; 5'-CATTTCTTGCAGAAAGCA-3', mutant; 5'-TGCTTTCTTCAAGAA ATG-3') were labelled with $\left[{ }^{32} \mathrm{P}\right]$ dCTP and hybridised to the membrane as described by Oldridge et al. ${ }^{8}$

\section{Methylation Studies}

To assess the pattern of $\mathrm{X}$-inactivation, methylation was analysed at the loci $P G K 1$ and $D X S 255$. Methylation studies were carried out on DNA from female carriers digested with the isoschizomers MspI (methylation insensitive) and HpaII (methylation sensitive) and probed either with pSPT-PGK (PGK1) or M27 $\beta$ (DXS255) as previously described. ${ }^{3}$

\section{Results}

\section{Pedigree 11}

The mutation in the affected boys is $961 \mathrm{G}>\mathrm{A}$ which in this case is not in the context of a highly mutable $\mathrm{CpG}$

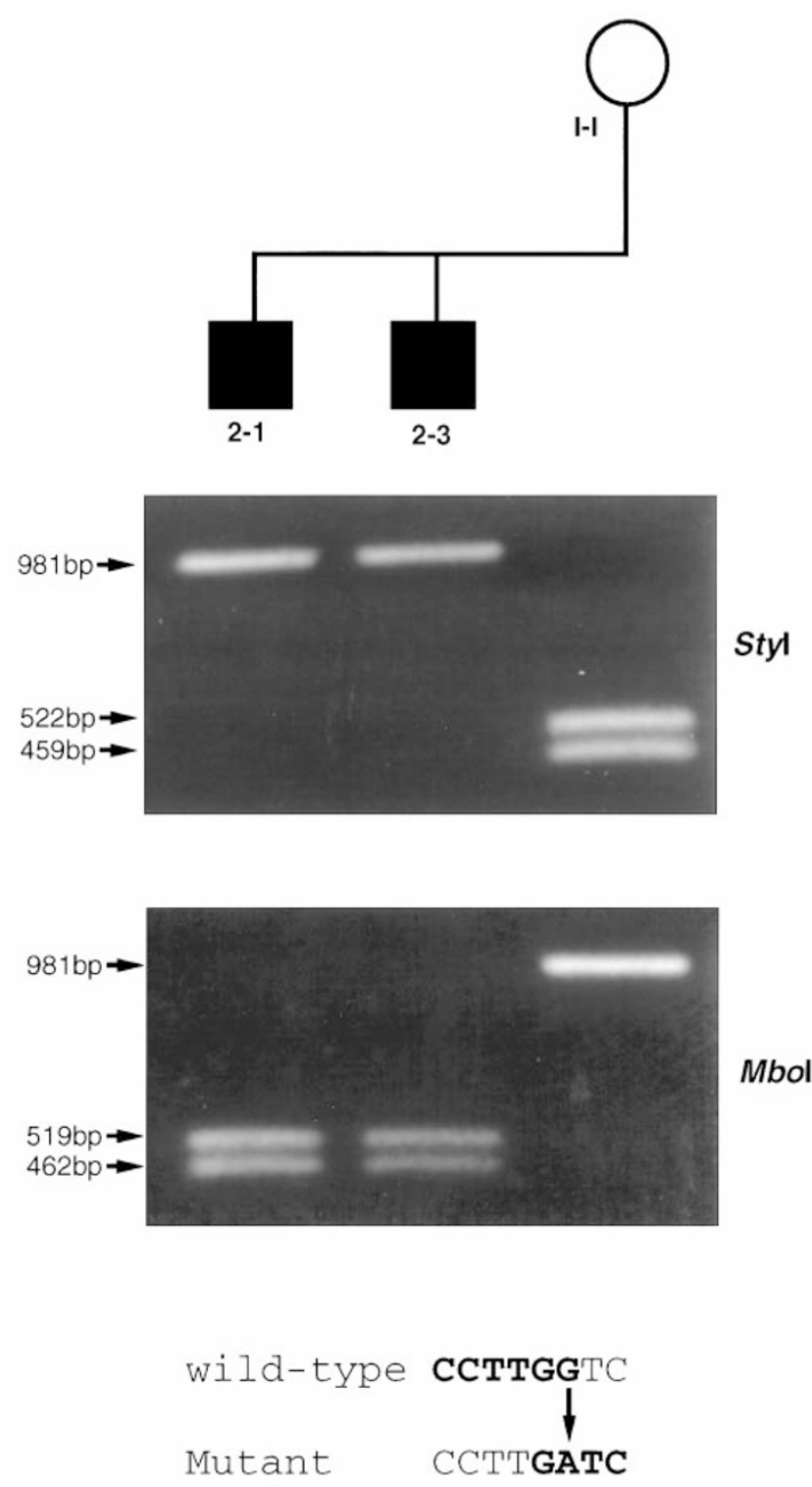

Figure 1 Restriction enzyme analysis of the 981bp xnp127/ PHD1 PCR product in pedigree 11. The affected boys are shown by closed squares, the mother by an open circle. Digestion with StyI cleaves the wild-type fragment; digestion with $\mathrm{MboI}$ cleaves the mutant fragment. In the lower figure, the context of the $G \rightarrow A$ mutation is shown; the enzyme sites are in bold 
sequence (Figure 1). The mutation destroys a StyI restriction site (CCTTGG) and creates an MboI site (GATC). This mutation, however, was absent in DNA from the mother's leukocytes (Figure 1) and buccal cells (data not shown). Serial dilutions were used to show that, by this method, the mutation would be detectable at 1 part in 64 .

\section{Pedigree 19}

Haplotype analysis demonstrated that the affected patient's sisters (2-2 and 2-3) had inherited the diseaseassociated haplotype (Figure 2). The pattern of $\mathrm{X}$ inactivation was determined by studying methylation status at loci DXS255 (for 1-1 and 2-2) and PGK1 (for 2-3). Using this analysis, we have previously shown that carriers of ATR-X have a skewed pattern of $\mathrm{X}$ inactivation ( $>90: 10$ ) with the mutant $X$ predominantly inactivated. In the females studied here we showed balanced patterns of $X$ inactivation in 1-1 and 2-2 but moderately skewed $X$ inactivation (about 80:20) in 2-3. However, in this case, contrary to the pattern found in ATR-X carriers, the $\mathrm{X}$ chromosome with the diseaseassociated haplotype was predominately active (data not shown).

No red cells with $\mathrm{HbH}$ inclusions were identified in the three females. On the basis of these findings it seemed likely that the mutation in the affected male occurred as a de novo event. Direct mutation analysis (Figure 3), however, revealed a weak positive signal for

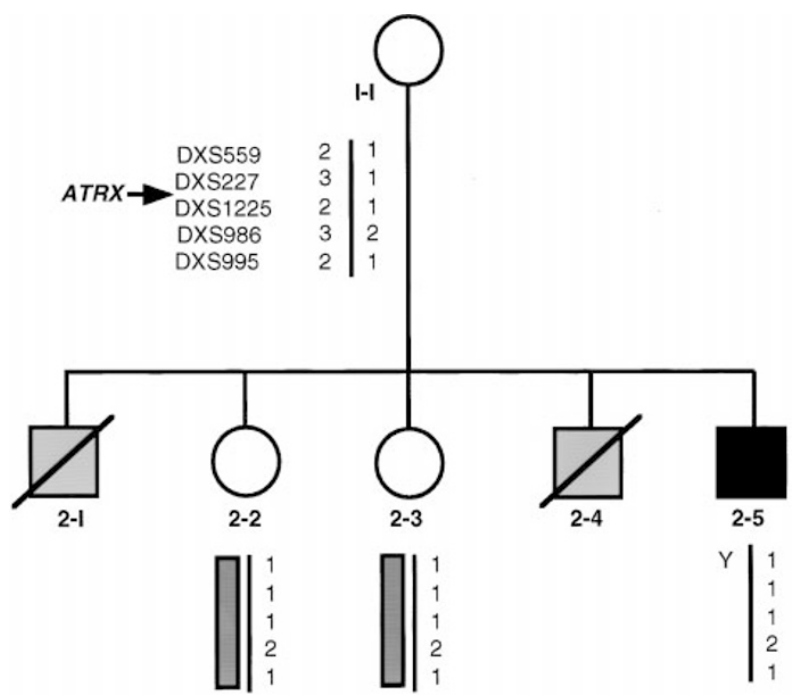

Figure 2 Pedigree 19 showing haplotypes: the order of the loci from centomere to Xqter is DXS559, DXS227, ATRX, DXS1225, DXS986, DXS995. The two male sibs, who were born prematurely and died soon after birth are indicated by shaded squares the mother (1-1) although both sisters (2-2, 2-3) were negative for the mutation. Serial dilutions showed that the signal in the mother was $1 / 64$ of that in the proband (2-5). Repeated analysis with fresh dilutions of the mother's DNA sample gave identical results. The possibility of contamination of the maternal DNA sample with the patient's was considered unlikely since the DNA extraction of blood samples had been carried out on separate occasions. A fresh maternal sample was not available.

\section{Discussion}

Two families are presented with evidence of maternal mosaicism for a mutation in $A T R X$. In pedigree 11, the mother has two affected boys but the underlying mutation is undetectable in two somatic tissues tested (leukocytes and buccal cells) and it is possible that the mosaicism is restricted to the germline. In contrast, in pedigree 19 , a mutation is detectable in the mother but absent from two sisters who share their affected brother's disease haplotype but appear not to carry the disease. This indicates that the mother is a gonosomal mosaic.

We have identified mutations in 60 families; three involve $>1 \mathrm{~kb}$ deletions, three involve insertions or

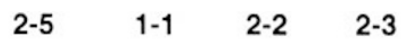

Wild-type probe
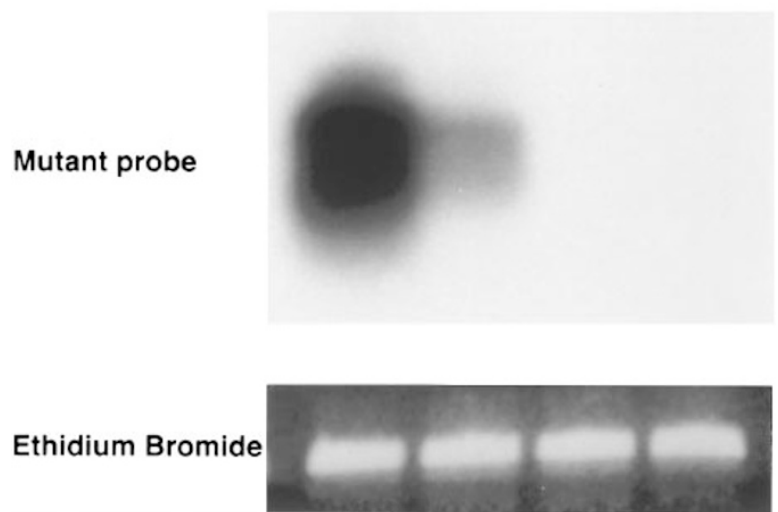

Figure 3 Allele specific oligo hybridisation using either wildtype or mutant specific probes against amplified DNA from pedigree 19 (see Figure 2). In the lower figure is the original gel, stained with ethidium bromide, to show equivalence of loading 
deletions of 1-2 bps, 54 are single base substitutions (manuscript in preparation). In 23 families there are affected males in different generations or branches of the pedigree and it is clear that the mothers of the probands are obligate carriers. In six cases material from the mother is not available for testing. In 31 families, where there is a sporadic case (20 families) or affected sibs (11 families), the mothers have all been tested for the presence of the mutation (summarised in Figure 4). There is evidence of maternal mosaicism in two families. Considering that many of the families in this study are small or have unaffected children too young for genetic testing, these figures may underestimate the frequency of mosaicism. For example, in singleton families the presence of germline mosaicism would not be apparent.

The principal issue when counselling is the risk of recurrence for families with a sporadic case of ATR-X. In this study, 17/20 mothers of sporadic cases were shown to be carriers of ATR-X by mutation analysis and this high figure may reflect a higher male than female mutation rate. Based on this experience, in families where the mutation has not been identified, we would expect $85 \%(17 / 20)$ of the mothers of sporadic cases to be carriers. Additional information may be sought by examining for the presence of $\mathrm{HbH}$ inclusions and studying the pattern of $\mathrm{X}$ inactivation (see above). If the mutation has been identified, then based on this small set of data, the risk of germline carriage in a mother testing negative for the mutation is $25 \%$ $(1 / 4)$.

In diseases where new mutations are frequent it is important, particularly for counselling the parents of sporadic cases, to know the likelihood of germline mosaicism. Zlotogora, in a review of the literature, ${ }^{9}$ distinguished two groups of diseases: group 1 in which somatic and germline mosaicism in one of the unaf-

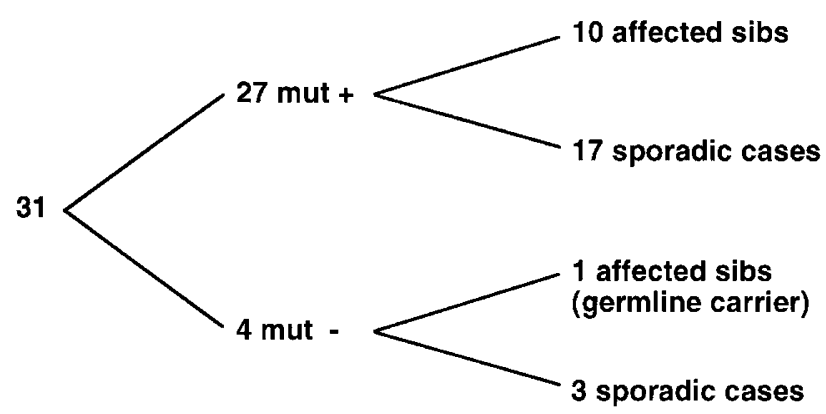

Figure 4 Summary of sporadic cases and affected sibs with ATR-X syndrome and the breakdown of cases born to mothers who test positive $(m u t+)$ or negative (mut-) for an ATRX mutation fected parents is relatively frequent, and group 2 in which mosaisicm is almost never found. ATR-X joins the group of conditions, eg fascioscapulohumeral dystrophy, haemophilia, Christmas disease, osteogenesis imperfecta and Duchenne muscular dystrophy, where post-zygotic mutation is relatively 'common's and germline mosaicism is an important consideration in the counselling of parents of sporadic cases when the mutation is not detected in the mother.

\section{Acknowledgements}

We are grateful to Dr DR Higgs and Dr AOM Wilkie for their helpful comments on the manuscript. We also thank the families for their cooperation and the clinicians who referred patients. This work was funded by the Wellcome Trust (042152).

\section{References}

1 Gibbons RJ, Brueton L, Buckle VJ et al: The clinical and hematological features of the X-linked $\alpha$ thalassemia/ mental retardation syndrome (ATR-X). Am J Med Genet 1995; 55: 288-299.

2 Donnai D, Clayton-Smith J, Gibbons RJ, Higgs DR: $\alpha$ thalassaemia/mental retardation syndrome (non-deletion type): report of a family supporting $\mathrm{X}$ linked inheritance. $J$ Med Genet 1991; 28: 734-737.

3 Gibbons RJ, Suthers GK, Wilkie AOM, Buckle VJ, Higgs DR: X-linked $\alpha$ thalassemia/mental retardation (ATR-X) syndrome: Localisation to $\mathrm{Xq} 12-21.31$ by $\mathrm{X}$-inactivation and linkage analysis. Am J Hum Genet 1992; 51: 1136-1149.

4 Gibbons RJ, Picketts DJ, Villard L, Higgs DR: Mutations in a putative global transcriptional regulator cause $\mathrm{X}$-linked mental retardation with $\alpha$-thalassemia (ATR-X syndrome). Cell 1995; 80: 837-845.

5 Gibbons RJ, Bachoo S, Picketts DJ et al: Mutations in a transcriptional regulator (hATRX) establish the functional significance of a PHD-like domain. Nat Genet 1997; 17: 146-148.

6 Picketts DJ, Higgs DR, Bachoo S, Blake DJ, Quarrell OWJ, Gibbons RJ: ATRX encodes a novel member of the SNF2 family of proteins: mutations point to a common mechanism underlying the ATR-X syndrome. Hum Mol Genet 1996; 5: 1899-1907.

7 Thein SL, Wallace RB: The use of synthetic oligonucleotides as specific hybridisation probes in the diagnosis of genetic disorders. In: Davies KE (ed). Human Genetic Diseases: a Practical Approach. IRL Press: Oxford, 1986, pp 33-50.

8 Oldridge M, Lunt PW, Zackai EH et al: Genotypephenotype correlation for nucleotide substitutions in the IgII-IgIII linker of FGFR2. Hum Mol Genet 1997; 6: 137-143.

9 Zlotogora J: Germ line mosaicism. Hum Genet 1998; 102: 381-386. 